\title{
Laserscribing of Thin Films Using Top-Hat Laser Beam Profiles
}

\author{
Stefan Rung , Mal Rexhepi*, Christian Bischoff** and Ralf Hellmann ${ }^{*}$ \\ *University of Applied Sciences Aschaffenburg, Applied laser and photonics group, \\ Wuerzburger Strasse 45, D-63743 Aschaffenburg \\ E-mail: stefan.rung@h-ab.de \\ ${ }^{* *}$ TOPAG Lasertechnik GmbH, Nieder-Ramstaedter Strasse 247, D-64285 Darmstadt \\ E-mail: bischoff@topag.de
}

\begin{abstract}
We present a comparative study of laser scribing different thin solid films using nanosecond laser pulses with different wavelengths and laser beam profiles. In particular, we show the influence of the used laser beam profile for the processing of a $150 \mathrm{~nm}$ thin Indium Tin Oxide transparent conductive film and a multilayer system, consisting of different metal and transparent conductive oxide (TCO) layers deposited on a soda lime glass substrate. For laser scribing the wavelengths $1064 \mathrm{~nm}$, $532 \mathrm{~nm}$ and $355 \mathrm{~nm}$ were used with a Gaussian and a Top-Hat beam profile. The homogenous TopHat beam profile is generated with both, a refractive and diffractive beam shaper. The results show several advantages of using a Top-Hat laser beam shape for thin film laser scribing, such as less energy consumption, high rim quality with simultaneous small pulse overlap and protection of the underlying substrate, respectively. Our results prove that the application of beam shapers can improve the process quality and increase the scribing speed for the investigated materials.
\end{abstract}

DOI: $10.2961 /$ jlmn.2013.03.0021

Keywords: Laser scribing, thin film, laser beam shaping, Top-Hat, ITO, TCO

\section{Introduction}

Within contemporary thin film processing, the laser has become indispensable as a tool for scribing and modification of a wide range of materials. A topic of upmost importance in this field is the structuring of transparent conductive films in the production process of thin film solar cells, touchscreens, display devices and organic light emitting diodes (OLEDs). Despite the recent progress in thin film ablation using ultra-short pulse lasers with pulse lengths in the femtosecond to picosecond regime [1-3], in industrial environments the most common laser source for these applications, particularly in solar cell production, is the pulsed solid state laser with nanosecond pulses. Alternative methods for structuring thin films are, e.g., scratching with needles or chemical etching. Compared with these techniques, laser scribing has the advantages of inexistent mechanical stress imposed to the treated materials and no hazardous substances are necessary for the process.

During the laser scribing process of thin films it is challenging to keep the heat-affected zone (HAZ) and the burr formation along the laser scribed grove as small as possible to guarantee a high quality laser ablation and not to hamper subsequent process steps or device performance. The HAZ is mainly determined by thermal material parameters as the thermal diffusion length and specific heat, thermomechanical properties, as well as by the laser wavelength, pulse length and pulse energy, respectively. Within the heat affected zone absorption of laser energy below the ablation threshold and thermal conduction can lead to a recast and crack formation. In addition, burr formation is related to the HAZ.
Hence, ultra-short pulse lasers have preferentially to be applied for laser scribing in case of OLED devices as they are associated with a reduced HAZ, being referred to as a cold ablation [4-5]An alternative approach is to alter the beam profile as for many applications a Gaussian beam does not lead to the best result. The main defiance in using Gaussian beam profiles is to manage the energy which is deposited into the material but not contributing to the ablation process. Figure 1 illustrates the principle of the ablation behavior of thin films. Complete ablation is achieved in an area where the laser intensity exceeds the ablation threshold. Any energy above this threshold may damages the underlying material. The energy in the flanks of the Gaussian profile is below the ablation threshold and leads to an undesired heating of the surrounding material. According to $\mathrm{Du}$, only up to $36.8 \%$ of the energy within a Gaussian beam is effectively used for the ablation of thin layers [6].

A further advantage of using a beam shaper to generate a Top-Hat profile is the possibility to change the laser footprint from a circular to a square or a rectangular one. Typically, structuring with a pulsed laser having a circular footprint leads to a saw-tooth type pattern along the ablated line which can induce unwanted mechanical stress in the thin film. To avoid this, it is necessary to use a high pulse overlap of at least $70 \%$ when working with a circular Gaussian beam profile [7]. In contrast, a rectangular footprint can produce straight walls with a very small overlap and thus increases the processing speed of the laser micromachining [8-10]. 
a)

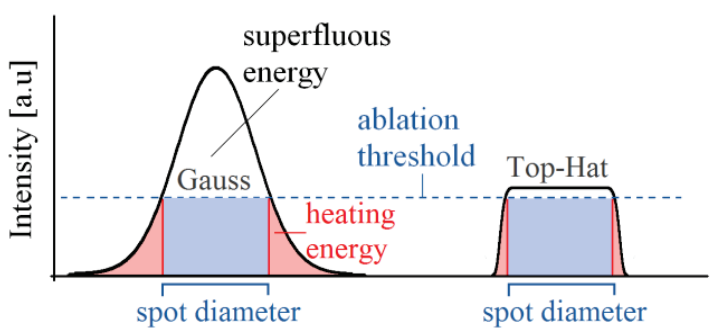

b)
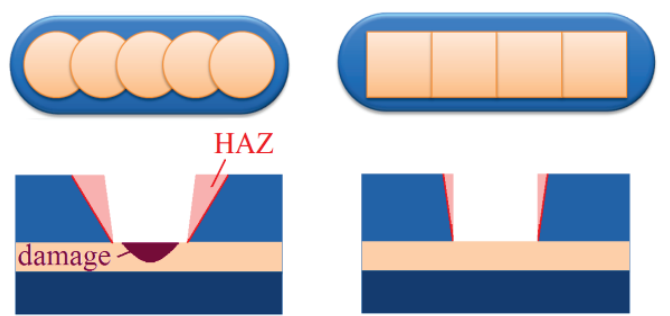

c)

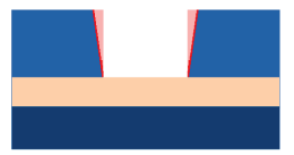

Figure 1: Different ablation behavior of thin films for Gaussian (left) and Top-Hat (right) laser beam profiles. a) cross section of laser beam profiles, b) ablated line with circular and rectangular laser foot print, c) schematic cross section of processed material system

\section{Experimental}

\subsection{Laser system}

We employed a $15 \mathrm{~W}$, pulsed Nd:YVO4 laser at 1064nm (EKSPLA Techno35C). By frequency conversion it is possible to generate the second harmonic $(532 \mathrm{~nm})$, third harmonic $(355 \mathrm{~nm})$ and the fourth harmonic $(266 \mathrm{~nm})$. The pulse duration can be varied between 6 and 20 ns depending on pump current and repetition rate $(2.5-100$ $\mathrm{kHz}$ ). The laser beam propagates with a $\mathrm{TEM}_{00}$ profile having a $\mathrm{M}^{2}<1.5$. Linear stages in $\mathrm{x}$ - and $\mathrm{y}$-direction provide an accuracy of $\pm 1 \mu \mathrm{m}$ and a travel speed of up to $500 \mathrm{~mm} / \mathrm{s}$ to move the samples with respect to the laser beam. The laser is focused using a lens system with a focal length of $50 \mathrm{~mm}$ that is mounted on another drive in z-direction.

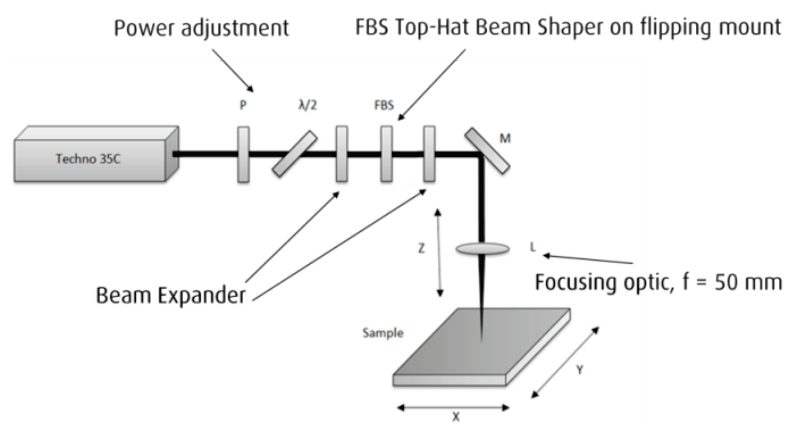

Figure 2: Illustration of the optical setup with the external attenuation based on a $\lambda / 2$ wave plate and a polarizer $(\mathrm{P})$. The Focus Beam Shaper (FBS) is situated in a beam expander. M: mirror, L: lens.

As can be seen in Figure 2, a combination of a rotatable polarizer and a half wave plate (HWP) is used for power adjustment. The Galilean-telescope is used to obtain a small focus size and secondly to adapt the beam size to the beam shaper size. For experiments with the Focus Beam
Shaper (FBS) the beam shaper is placed inside the telescope and for studies with the Gaussian to Top-Hat beam shaper (GTH) the beam size in front of the telescope is sufficient.

\subsection{Beam Shaping Technique}

One kind of Top-Hat beam shaping elements is based on so called mapping concepts [11]. Using these allows calculating a certain deflection angle for each point on the optical surface and leads to aspheric optical elements. Each element requires a certain size of input beam and intensity distribution, typically single-mode Gaussian. These preadapted beams have to be centered to the beam shaping element. Typical tolerances for variation of input beam size and lateral misalignment are $5-10 \%$, of the used beam diameter. Refractive versions of such a beam mapper show a shaping efficiency $>95 \%$. The smallest achievable Top-Hat spot size is roughly 4 times larger than the diffraction limited unshaped Gaussian spot size, with regard to $1 / \mathrm{e}^{2}-$ limit.

Previously reported studies on thin film processing with Top-Hat profiles typically used systems based on beam mapping concepts to generate the beam profile $[7,8,12]$. These systems consist of several optical elements and combine partly optical functions like adaptation of input beam size and focussing in one housing. This can lead to a higher sensitivity especially to angle adjustment or lateral displacement. Due to the bigger size of such systems the integration of beam shaping into existing laser systems can be more complicated.

In this study two different kinds of commercially available beam shapers are used ${ }^{1}$. The GTH-beam shaper is based on refractive mapping concepts. The Focus Beam Shaper (FBS) is based on a new diffractive beam shaping concept realized by smooth phase-modulating height profiles. Both beam shapers, GTH and FBS, consists of just one single element. This allows a very easy alignment and integration into existing optical setups. By introducing these beam shapers into the beam path the diffraction limited Gaussian spot in the focal plane is transformed into a square Top-Hat beam profile. The user just has to consider using the correct adapted beam size at the position of the beam shaping element. This can easily be realized by putting the beam shaping elements into a beam expander as shown in this study.

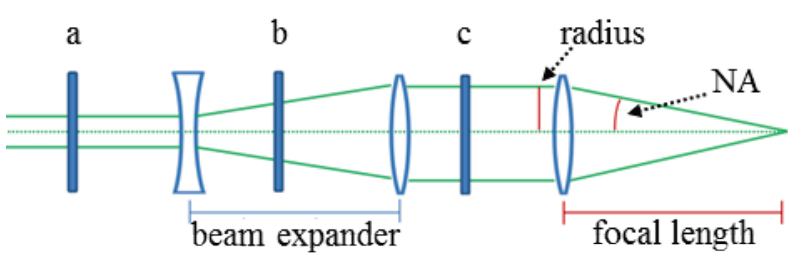

Figure 3: Possible positions for integration of GTH and FBS beam shaper into beam path: a) beam shaper before beam expander, b) beam shaper in beam expander, c) beam shaper behind beam expander.

The diffractive beam shaper FBS has a shaping efficiency of $>95 \%$ and a uniformity of $\pm 2.5 \%$ of the Top-Hat

\footnotetext{
${ }^{1}$ www.topag.de
} 
plateau $[13,14]$. Using the FBS a Top-Hat size of approx. 1.5-fold of the regular Gaussian size can be generated.

For the beam shapers used here, the achievable Top-Hat size $\Phi$ at the focal plane is mainly determined by the numerical aperture NA of the focused beam as shown in figure 3. The effective size can be calculated as follows:

$$
\begin{aligned}
\Phi_{F B S} & =\frac{\lambda}{N A}=\frac{\lambda \cdot \text { focal length }}{\text { beam radius }\left(1 / e^{2}\right)} \\
\Phi_{G T H} & =\frac{3.6 \mu m}{N A}=\frac{3.6 \mu m \cdot \text { focal length }}{\text { beam radius }\left(1 / e^{2}\right)}
\end{aligned}
$$

\subsection{Material Setup}

Laser patterning experiments were performed on two different thin film systems. On the one hand we use a $150 \mathrm{~nm}$ thin Indium Tin Oxide layer deposited on soda lime glass. This is a typical film thickness for OLED applications, the laser patterning process of which has been extensively investigated by several research groups (5, 15-18). However, these studies employed Gaussian beam profiles with the impact of different beam shapes being unexplored. Contrary to these publications, in this contribution we focus on the optimization of the patterning process with respect to scribing efficiency and scribing speed by using Top-Hat profiles.

Furthermore, a multilayer system of different TCO and metal layers out of the OLED production is used. The order of the layer stack from bottom to top is $\mathrm{SiO}_{2}$, ITO, compounds of Molybdenum, Aluminum and Molybdenum, respectively. Metalized ITO structures are used to enhance the conductivity of the conductive oxide layer in device zones in which the conductivity of the TCO is insufficient. The total height of the five layers is about $740 \mathrm{~nm}$, being deposited on a soda lime glass substrate. To illustrate this multilayer setup, a schematic drawing is shown in figure $7 \mathrm{~b}$. In contrast to pure ITO layers, the patterning of such multi-layer setups has hardly been reported before. Due to the significant different thermal properties of each layer the common patterning process of this setup is wet etching. Former studies of our group show, using a ns-laser system with a Gaussian beam profile causes a large HAZ which leads to distinct burr formation and a huge amount of debris on the surrounding surface $[10,19]$. Due to the bad quality it isn't applicable for manufacturing processes.

\subsection{Experimental procedure and analysis}

The main focus of the first part of the study is to improve the scribing efficiency of the ITO patterning according to scribing speed and energy consumption. Hence, it is our objective to combine high quality laser scribing with small pulse to pulse overlap and minimized energy efforts. The influence of the process parameters pulse energy, pulse to pulse overlap and direction of scribing (the laser impinging the structure from the top or through the substrate) for the wavelength $1064 \mathrm{~nm}$ were investigated while using a FBS Top-Hat beam shaper.

The second part of the experimental work, the processing of the metallized ITO layer, was performed with both, a refractive (GTH) and a diffractive (FBS) Top-Hat beam shaper. For the second and the third harmonic wave- length of the laser the influences of pulse energy, pulse to pulse overlap, repetition rate and direction of scribing are analyzed.

The experimental procedure with both materials is similar. In the first step it is necessary to align the beam shapers which will be controlled via single pulse ablation in the appropriate material. Once a well-defined rectangular ablation spot can be detected, the line ablation can be performed.

Patterning with the Top-Hat profile is carried out from both the TCO and the substrate side. The requirements on the laser scribed lines are full electrical isolation by the generated groove, a plane and clean bottom of the groove and the shoulders at the rim should be minimized.

To compare the different laser scribing approaches several criteria were monitored. Besides the mandatory electrical isolation of the trenches which is measured with a digital multimeter, the burr volume and the roughness of the line's bottom are determined with the laser scanning microscope VK-X200 from Keyence and the confocal microscope CFM from FRT. The determination of the burr volume is carried out with a software based analyzing tool of the microscopes, for which the user defines a reference surface level and the material volume inside a defined area which is higher than this reference level is calculated.

Studies for the scribing of the multi-layer system with the FBS beam shaper require an additional measurement value to compare the influence of several process parameters and give further information about the expansion of the heat-affected zone. With the measured ablation diameter of the top and the bottom of the film stack and the given total height of $740 \mathrm{~nm}$, the angle of the rims is calculated. A lager angle indicates a distinctive HAZ due to the high amount of molten metal from the top films.

\section{Results and Discussion}

\subsection{Laserscribing of Indium Tin Oxide}

The comparative values with the Gaussian beam profile were identified in previous studies [20] from the TCO side. We define high ablation quality by three factors: i) a small burr formation and ii) a homogenous material ablation at the bottom of the line which can be indicated through a small roughness along the trench. These features are analyzed by a confocal laser scanning microscope. The third quality factor is the straightness of the walls without a distinctive saw-tooth pattern at the rims. The volume of the generated burr is analyzed by using a software tool of the laser scanning microscope that calculates the material volume within given height limits. By setting the lower height limit to the surface, the calculation delivers the volume of the generated burr.

For the comparative study, initially the influence of the repetition rate $(2.5-40 \mathrm{kHz})$, pulse energy $(4-55 \mu \mathrm{J})$ and pulse to pulse overlap $(15-90 \%)$ on the ablation quality has been examined for a Gaussian beam profile. Figure 4 shows the best result of the line ablation. The trench which is generated with a repetition rate of $10 \mathrm{kHz}$, a pulse energy of $11 \mu \mathrm{J}$ and an overlap of $90 \%$ has straight walls and a smooth bottom with a roughness of $\mathrm{Ra}=5 \mathrm{~nm}$. Within the area of $180 \mu \mathrm{m} \times 30 \mu \mathrm{m}$ a burr volume of $9.3 \mu \mathrm{m}^{3}$ can be measured. This result confirms the possibility of a high 
quality laser scribe using a Gaussian beam profile, yet at a very high pulse overlap of $90 \%$.

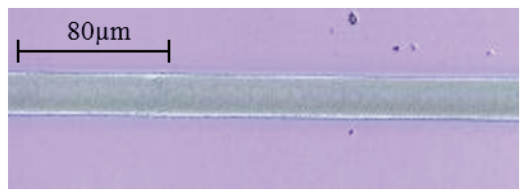

b)

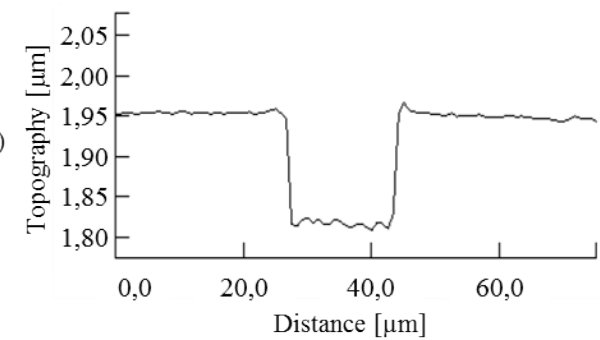

Figure 4: (a) Microscope picture and (b) Cross section of the generated trench in $150 \mathrm{~nm}$ ITO film generated with the optimized parameter set using a Gaussian beam profile at $1064 \mathrm{~nm}$, pulse energy $11 \mu \mathrm{J}$, pulse repetition rate $10 \mathrm{kHz}$, overlap rate $90 \%$, pulse duration $7 \mathrm{~ns}$, laser impinging from substrate or TCO side

Figure 5 compares the laser scribing results for Gaussian and Top Hat beam profiles. The diagram shows the burr volume as a function of pulse overlap for the Gaussian beam profile (blue/solid), the film side performed Top-Hat (green/dashed) scribe and the substrate side performed TopHat scribe (red/dashed-dotted), respectively. In case of the Gaussian beam profile, the burr volume increases clearly with decreasing pulse overlap. When using a pulse overlap greater than $60 \%$ the generated burr volume of both the Top-Hat and Gaussian beam profile are comparable. However, for laser scribing using a Top-Hat beam profile with a pulse overlap smaller than $60 \%$, the burr volume is significantly lower as compared to the case of using a Gaussian profile. In addition, the burr volume remains on a lower level when the pulse overlap is further decreased. Moreover, in case the ablation is performed from substrate side, the burr volume even decreases with decreasing pulse overlap. The smallest burr volume $\left(13 \mu \mathrm{m}^{3}\right)$ when using a TopHat profile results from processing through the substrate at $20 \mathrm{kHz}$ repetition rate and a pulse energy of $13 \mu \mathrm{J}$ with a very small overlap of $8 \%$. In this case, the groove has still straight walls and a good bottom roughness of $\mathrm{Ra}=10 \mathrm{~nm}$. This significantly reduced pulse overlap corresponds to an enhancement of the processing speed of about $900 \%$ as compared to the Gaussian beam [3,5] while still achieving a comparable quality.

\subsection{Laserscribing of metallized Indium Tin Oxide}

In former unreported experiments of our workgroup with the metallized ITO system and Gaussian beam profiles, the wavelengths $532 \mathrm{~nm}$ and $355 \mathrm{~nm}$ showed the most promising results for further studies. First the diffractive beam shaper FBS is applied for the visible laser beam at $532 \mathrm{~nm}$. Here an almost diffraction limited spot size of approximately $7 \mu \mathrm{m}\left(1 / \mathrm{e}^{2}\right)$ is used. For experiments with the third harmonic of our laser system, the refractive beam shaper GTH is installed and produces a rectangular laser footprint with an edge length of $68 \mu \mathrm{m}$.

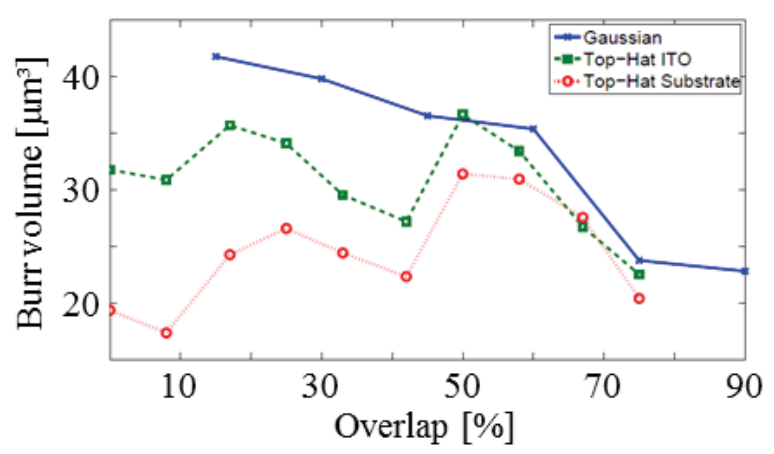

Figure 5: Comparison of burr volume as a function of overlap for the line ablation of $150 \mathrm{~nm}$ thin ITO films at $1064 \mathrm{~nm}$ with a Gaussian and a Top-Hat (FBS) beam profile

\section{Beam shaping by FBS beam shaper at $532 \mathrm{~nm}$}

By the fact that the previous accomplished Gaussian experiments and the now performed investigations with the FBS beam shaper generated similar line widths, the results can directly compared regarding to similar dimensions of ablated material volume. Best results with the Gaussian profile have been obtained if the material has been processed from the substrate side at a laser power of $50 \mathrm{~mW}$ (10ns pulses at $20 \mathrm{kHz}$ ). With a pulse overlap of $60 \%$, the best compromise between smooth walls and small burr formation was found. Through the application of the diffractive optical element (DOE) the original round laser footprint can be transform into a rectangular shape. The ablation of single pulses shown in figure 6 reflects the laser footprint and the homogenous ablation depth. The surrounding HAZ is reduced to a minimum. Hence, the burr formation and the debris are hardly present.

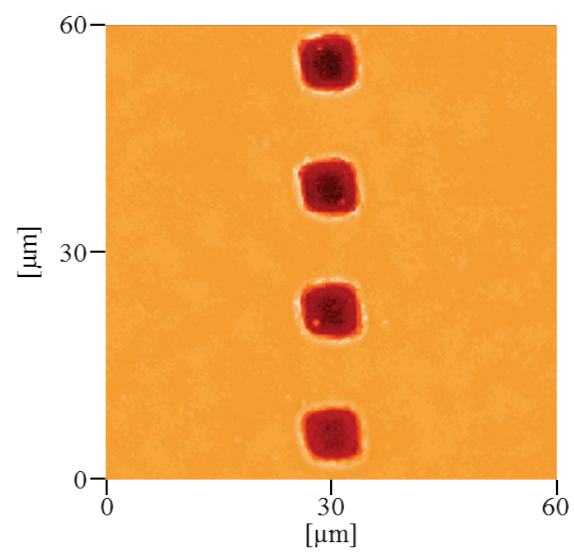

Figure 6: 2-dim. false color representation of generated separated rectangular ablation spots using Top-Hat shaper FBS at $532 \mathrm{~nm}$, laser power $36 \mathrm{~mW}$, repetition rate $20 \mathrm{kHz}$, pulse duration $10 \mathrm{~ns}$, laser impinging from substrate or TCO side

In order to achieve smooth side walls and a straight trench without a saw tooth pattern, both the pulse to pulse overlap and the laser power can be significantly reduced to $8 \%$ and $18 \mathrm{~mW}$, respectively, when using the FBS beam 
shaper. The reduction of pulse to pulse overlap corresponds to an increase of processing speed at comparable system conditions. In relation to the processing parameters for line ablation with the Gaussian beam profile the processing speed can be more than doubled. In addition, the lower laser power and Top-Hat beam profile are associated with an undamaged substrate, less burr formation and debris along the laser generated trench. Figure 7 shows a 2D plot of the ablated trench. Along the rims some scattered burr formations can be detected. The cross section of the line demonstrates the integrity of the underlying substrate.

a)

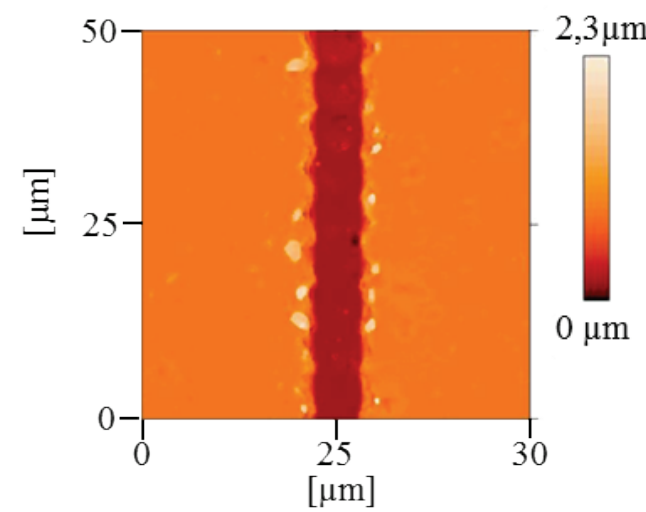

b)

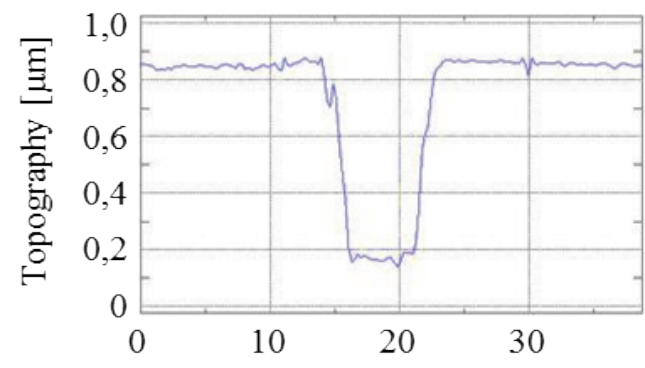

Distance $[\mu \mathrm{m}]$

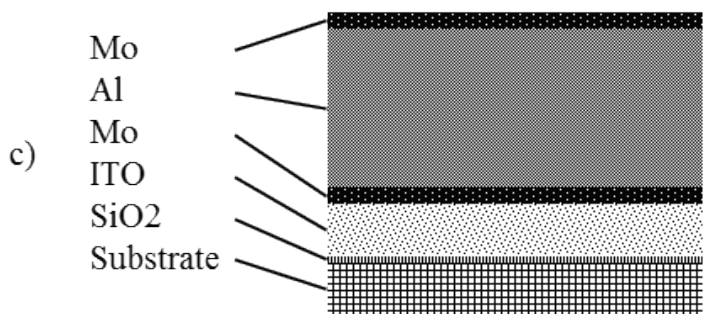

Figure 7: (a) 2-dim. false color representation and (b) cross section of generated trench using Top-Hat shaper FBS at $532 \mathrm{~nm}$, laser power $18 \mathrm{~mW}$, repetition rate $20 \mathrm{kHz}$, overlap rate $8 \%$, laser impinging from substrate or TCO side. (c) The material setup of metallized ITO system

\section{Beam shaping by GTH beam shaper at $355 \mathrm{~nm}$}

The results using the FBS beam shaper showed that a significant improvement of the laser scribing can be achieved as compared to a Gaussian beam profile in both productivity and quality. To demonstrate further benefits in using a Top-Hat beam profile a refractive GTH beam shaping lens is employed in combination with the UV at 355 $\mathrm{nm}$. Laser scribing of transparent conductive oxides in the UV might be advantageous due to the high absorption of the TCO and the potential reduction of thermal effects in this spectral range. However, the high absorption of the substrate may lead to a substrate damage hampering device performance. As a result, the process window ensuring a high quality laser ablation of a TCO in this spectral range might be limited. The usage of a homogenous beam profile, however, can enlarge this process window and make UV laser light more attractive in thin film patterning.

The GTH beam shaping lens used in this study at 355 nm generates a homogenous beam profile with an edge length of $65 \mu \mathrm{m}\left(1 / \mathrm{e}^{2}\right)$ and with the resulting beam shape being less dependent from possible deviations from a perfect input beam (e.g. $\mathrm{M}^{2}$ or ellipticity). Figure 8 demonstrates the almost square ablation shape with a homogenous ablation depth. The small burr formation at the left rim can be neglected, as a horizontal processing direction is chosen.

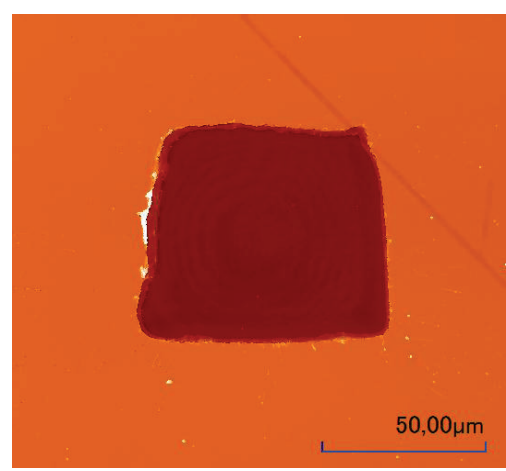

Figure 8: 2-dim. false color representation of generated separated rectangular ablation spots using Top-Hat shaper GTH at $355 \mathrm{~nm}$, laser power $170 \mathrm{~mW}$, repetition rate $2.5 \mathrm{kHz}$, pulse duration $6 \mathrm{~ns}$, laser impinging from substrate or TCO side

Using an overlap between $6 \%$ and $50 \%$ generates a burr volume between approx. $100 \mu \mathrm{m}^{3}$ and $950 \mu \mathrm{m}^{3}$. Investigations with an overlap higher than $50 \%$ show a steady increase of burr volume caused by the larger HAZ. For the calculation of the burr volume an area of $170 \mu \mathrm{m}^{*} 170 \mu \mathrm{m}$ is considered. Figure 9 shows the influence of the overlap to the burr volume. The bars in the chart indicate the influence of the used laser power $(80-380 \mathrm{~mW})$ and repetition rates $(2.5 \mathrm{kHz}-20 \mathrm{kHz})$ for these studies.

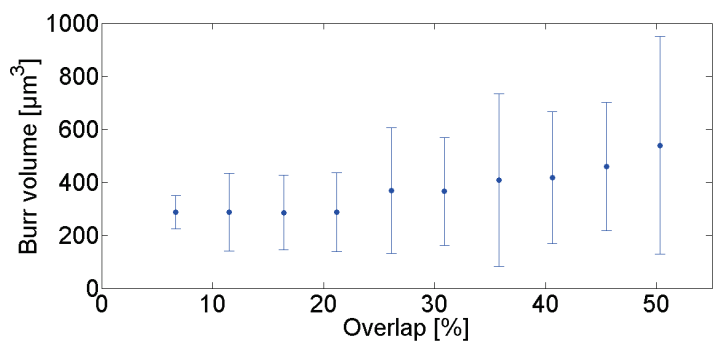

Figure 9: Comparison of burr volume as a function of overlap between $6 \%$ and $50 \%$ for line ablation in metallized ITO with GTH beam shaper at $355 \mathrm{~nm}$, repetition rate $2.5 \mathrm{kHz}$, bars show the influence of the laser power 
With a pulse to pulse overlap of $16 \%$ and $170 \mathrm{~mW}(2.5$ $\mathrm{kHz}$ ) a trench is scribed into the metallized TCO film and generates an insulating line without any visible debris, less burr formation at the rim and no substrate damage (figure 10). The bottom of the line shows a homogenous removal of the material.

In comparison with the investigations using the FBS beam shaper at $532 \mathrm{~nm}$ the ablation quality of the Top-Hat patterning with GTH beam shaper at $355 \mathrm{~nm}$ show better results regarding to burr formation.

a)

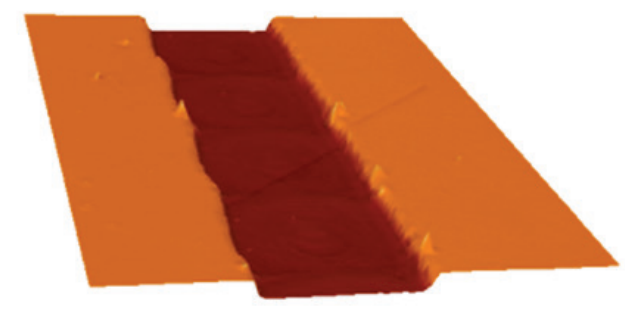

b)

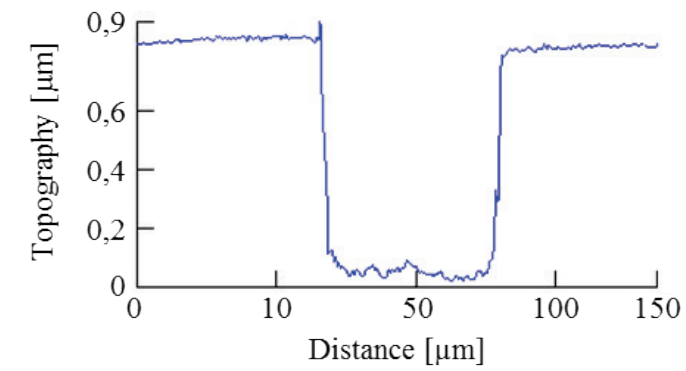

c)

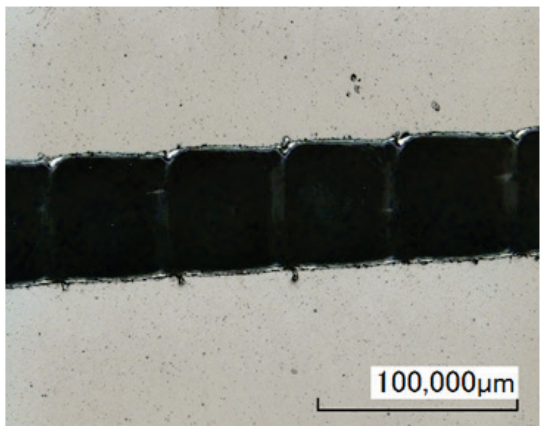

Figure 10: (a) 3-dim. false color representation, (b) cross section and (c) microscope picture of generated trench in metallized ITO setup using Top-Hat shaper GTH at $355 \mathrm{~nm}$; laser power $170 \mathrm{~mW}$, repetition rate $2.5 \mathrm{kHz}$, pulse overlap $16 \%$, pulse duration $6 \mathrm{~ns}$, laser impinging from substrate or TCO side

\section{Summary}

We present results of a comparative study of laser pattering of ITO and metallized ITO thin films using Top Hat beam shapers. We demonstrate that laser processing can be significantly improved by using a Gaussian-to-Top-Hat beam shaper. In particular, through the use of a uniform laser profile instead of a Gaussian beam profile the throughput of the structuring process of ITO thin films can be increases while achieving a comparable quality of the laser scribed lines. In this study, an increase of scribing speed of about $900 \%$ was achieved for laser patterning of thin ITO films. Material setups like the used metallized
ITO shows no acceptable results by processing with Gaussian beam profiles for nanosecond lasers. By the application of Top-Hat beam shapers it is possible to machine this multilayer system with satisfying process results. In this study the combination of the wavelength $355 \mathrm{~nm}$ and the refractive beam shaper GTH produce better results for line ablation for the metallized ITO setup. This show the importance of an application related choice of the process parameters with respect to wavelength, laser beam shape and the corresponding beam shaping method.

\section{References}

[1] G. Heise, M. Dickmann, M. Domke, A. Heiss, T.

Kuznikcki, J. Palm, I. Richter, H. Vogt and H. Huber: Appl. Phys A, 104 (2011), p.387.

[2] S. Xiao, B. Schöps and A. Ostendorf: Physics Procedia, 29 (2012), p.594.

[3] A. Risch and R. Hellmann: Physics Procedia, 12, (2011) p.133.

[4] G. Raciukaitis, M. Brikas, M. Gedvilas, T. Rakickas: Appl Surf Sci, 253/15 (2007), p.6570.

[5] A. Risch and R. Hellmann: Applied Surface Science, 258/5, (2011) p.1849.

[6] K. Du: SPIE Proc. Photonics West, (2009) 7202

[7] O. Homburg, F. Völkermeyer, F. Toennissen, H. Ganser, T. Mitra: Proc. Lasers in Manufacturing, Munich, (2007) p.754.

[8] B. Baird, T. Gerke, K Wieland and N. Paudel: Proc. 26th European Photovoltaic Solar Energy Conference, Hamburg, (2011) p.2471.

[9] C. Bischoff, E. Jäger, St. Rung and R.Hellmann: Laser Technik Journal, 5, (2012) p.15.

[10] S.Rung and R. Hellmann: Proc. 26th Int. Scientific Conference microCAD, Miskolc, (2012) D13.

[11] F. Dickey, L. Weichmann, R. Shagam: Proc. SPIE 4065, High-Power Laser Ablation III, (2000) 338.

[12]E. Steiger, M. Scharnagl, M. Kemnitzer and A. Laskin: Proc. ICALEO, Orlando, (2009) M1107.

[13] U. Umhofer, E. Jäger and C. Bischoff: Laser Technik Journal, 3, (2011) p.24.

[14] G. Raciukaitis, E. Stankevicius, P. Gecys, M. Gedvilas, C. Bischoff, E. Jäger, U. Umhofer and F. Völklein: JLMN, 6/1, (2011) p.37.

[15] S. Xiao, E.L. Gurevich and A. Ostendorf: Appl Phys A, 107 (2012), p.333.

[16] M. Henry, P.M. Harrison and J. Wendland: JLMN, 2 (2007), p.49.

[17]A. Schoonderbeek, V. Schütz, O. Haupt and U. Stute: JLMN, 5 (2010), p.248.

[18]T. Szörenyi, L.D. Laude, I. Bertoti, Z. Kantor and Z. Geretovszky: J Appl Phys, 78, (1995) p.6211.

[19] C. Bischoff, E. Jäger, S. Rung, and R. Hellmann: Proceedings of the LASYS - SLT, Stuttgart, (2013) p.57.

[20] S. Rung, M. Rexhepi and R. Hellmann: Proc. 27th Int. Scientific Conference microCAD, Miskolc, (2013) D3.

(Received: July 31, 2013, Accepted: November 24, 2013) 